\title{
Metabolic Syndrome Is Associated with Greater Histologic Severity, Higher Carbohydrate, and Lower Fat Diet in Patients with NAFLD
}

\author{
Hellan Kang, M.D., ${ }^{1}$ Joel K. Greenson, M.D., ${ }^{2}$ Jason T. Omo, B.A., ${ }^{1}$ Cewin Chao, M.S., R.D., ${ }^{3}$ \\ Debra Peterman, R.D., ${ }^{3}$ Lilian Anderson, M.S., R.D., ${ }^{3}$ Laura Foess-Wood, B.S., ${ }^{3}$ Mary A. Sherbondy, M.D., \\ M.S., ${ }^{1}$ and Hari S. Conjeevaram, M.D., M.S. ${ }^{1}$ \\ ${ }^{1}$ Division of Gastroenterology, Department of Medicine, ${ }^{2}$ Department of Pathology, and ${ }^{3}$ General Clinical \\ Research Center, The University of Michigan, Ann Arbor, Michigan
}

\begin{abstract}
OBJECTIVES: Nonalcoholic fatty liver disease (NAFLD) is considered as the hepatic manifestation of metabolic syndrome. Insulin resistance (IR) is a key component of metabolic syndrome. The aim was to determine the dietary composition, physical activity, and histologic severity between NAFLD patients with and without metabolic syndrome.

METHODS: $\quad$ Ninety-one patients with NAFLD completed the Block Food Frequency Questionnaire and the Paffenbarger Physical Activity Questionnaire. IR was assessed by the homeostasis model assessment (HOMA) index. Metabolic syndrome was defined by the ATP III clinical definition. Nonalcoholic steatohepatitis (NASH) Clinical Network Scoring System was used to determine the histologic severity of NAFLD.
\end{abstract}

RESULTS: $\quad$ Thirty-one patients (34\%) had metabolic syndrome. Patients with metabolic syndrome had a higher HOMA index ( 7.66 vs $4.45, p=0.04)$, and consumed more carbohydrates $(51 \%$ vs $45 \%, p=0.03)$ and less fat ( $34 \%$ vs $40 \%, p=0.01)$ compared with those without metabolic syndrome; total daily calorie, protein consumption, and physical activity were similar between the two groups. Patients with metabolic syndrome had higher scores for steatosis $(2.0 \pm 0.8$ vs $1.37 \pm 1, p=0.02)$, NASH activity $(4.13 \pm 1.4$ vs $3.13 \pm 1.7, p=0.004)$, and global NASH score $(5.9 \pm 1.7$ vs $4.4 \pm 2.3, p=$ $0.0006)$ compared with those without metabolic syndrome. When controlled for other factors including dietary composition and physical activity, the presence of metabolic syndrome was a significant risk factor for global NASH severity in addition to HOMA index and female gender.

CONCLUSION: Metabolic syndrome in patients with NAFLD is associated with a diet containing more carbohydrate and less fat and greater histologic severity. The role of a carbohydrate-restricted diet in decreasing the risk for metabolic syndrome and histologic severity should be assessed in patients with NAFLD.

(Am J Gastroenterol 2006;101:2247-2253)

\section{INTRODUCTION}

Nonalcoholic fatty liver disease (NAFLD) is now recognized as one of the most common forms of chronic liver disease in developed countries with an estimated prevalence of 10 $24 \%$ in the general population $(1,2)$. Obesity, type 2 diabetes mellitus (DM), and hyperlipidemia are common risk factors for NAFLD. As can be seen from the recent National Health and Nutrition Examination Survey (NHANES) data (19992002), the proportion of the U.S. population that is overweight or obese is on the rise, with $65.2 \%$ of the population being overweight (body mass index [BMI] $>25$ ) and $30.1 \%$ being obese (BMI >30) (3). With the increase in the prevalence of obesity in the United States and the association of obesity and NAFLD, the prevalence of NAFLD is expected to increase in the United States.
NAFLD is considered to be the hepatic manifestation of metabolic syndrome. Metabolic syndrome is also increasing in prevalence in the United States. It has increased from $23.1 \%$ in the NHANES III (1988-1994) study (4) to $26.7 \%$ in the recently published NHANES study (1999-2002) for adults aged 20-70 yr (3). Metabolic syndrome increases with age and is found more commonly in women.

Several recent studies have now shown that insulin resistance (IR) is a major step in the pathogenesis of NAFLD (5-9). It is also the pathogenic link underlying the metabolic abnormalities that constitute the metabolic syndrome such as obesity, diabetes, and dyslipidemia (10). Metabolic syndrome can be induced by different environmental factors, including dietary habits (11). Recent reports have shown that the adverse cardiovascular risk profile seen as part of metabolic syndrome is associated with a diet high in carbohydrate and 
low in unsaturated fat and also with decreased physical activity $(5,12,13)$. A recent study also showed that patients with NAFLD consuming a diet high in carbohydrates and low in fat have greater odds of hepatic inflammation (14), suggesting a link between diet and liver injury possibly mediated through IR. There is no study to date that has assessed the relationship among metabolic syndrome, dietary composition, physical activity, and histologic severity in patients with NAFLD.

The aims of this study were to determine dietary composition and physical activity and their relationship to histologic severity between NAFLD patients with and without metabolic syndrome.

\section{METHODS}

\section{Patients}

This was a prospective study of predictors of histologic severity in patients with NAFLD. Patients who presented to the Hepatology clinic at the University of Michigan Medical Center from March 2003 to November 2004 were enrolled into the study by the following inclusion criteria: Patients $12 \mathrm{yr}$ or older with clinically suspected NAFLD based on persistent elevation in aminotransferase levels ( $>1.5$ upper limit of normal on two or more occasions at least 3 months apart) and/or radiologic evidence of fatty liver with no identified etiology and alcohol consumption less than $40 \mathrm{~g} / \mathrm{wk}$. A liver biopsy should have been performed within $1 \mathrm{yr}$ of entry into the study and liver biopsy slides should be available for review before enrollment. Patients were excluded from the study for the following reasons: Other causes of chronic liver diseases, alcohol use more than $40 \mathrm{~g} / \mathrm{wk}$ ( 5 drinks/wk or more) within the past 3 months or a history of long-term alcohol abuse or dependence in the past, use of medications reported to cause steatosis (including amiodarone, steroids, tamoxifen, valproic acid, methotrexate, IV tetracycline) within the past 3 months or greater than 6 months in the past $2 \mathrm{yr}$, weight reduction surgery within $1 \mathrm{yr}$ or jejuno-ileal bypass in the past, weight loss medication or participation in weight loss program in the past 3 months, evidence of hepatic decompensation, HIV antibody positive, pregnancy, or breast feeding. The protocol and consent form were approved by the Institutional Review Board of the institution. All patients signed the consent form at the time of screening for the study.

\section{Study Design}

Clinical information regarding the patients' medical and surgical history as well as medications was recorded. Laboratory parameters to exclude other causes of chronic liver diseases were examined. All patients underwent anthropometric and laboratory investigations. Measurement of the waist and hip circumference, weight, height, BMI, and blood pressure was performed. Laboratory testing for metabolic abnormalities including fasting triglyceride, HDL, glucose, and insulin was obtained. Measurement of IR was determined by the home- ostasis model assessment (HOMA) index (HOMA index = [(fasting insulin $\times$ fasting glucose/18)/22.5]) (15). A HOMA index value of $\geq 2.0$ was selected to define IR in this study as this value is considered abnormal and indicative of decreased insulin sensitivity in normal range weight and obese individuals $(15,16)$.

Liver biopsy slides were obtained and reviewed by a pathologist. Patients who were enrolled completed the Block Food Frequency Questionnaire (FFQ 98) (17) and the Paffenbarger Physical Activity Questionnaire (PAQ) (18). The presence of metabolic syndrome was defined by the criteria proposed by the Third Report of National Cholesterol Education Program expert panel on detection, evaluation, and treatment of high blood cholesterol in adults (ATP III criteria) (19) as patients who have three or more of the following: (1) Central obesity measured by waist circumference greater than 35 inches in women and 40 inches in men, (2) blood pressure greater than or equal to $135 / 85 \mathrm{mmHg}$, (3) fasting glucose greater than or equal to $110 \mathrm{mg} / \mathrm{dL}$, (4) fasting triglyceride greater than or equal to $150 \mathrm{mg} / \mathrm{dL}$, and (5) HDL less than $50 \mathrm{mg} / \mathrm{dL}$ in women and $40 \mathrm{mg} / \mathrm{dL}$ in men. Patients who were being treated with antihypertensive, lipid lowering, and diabetic medications were considered as having hypertension, hyperlipidemia, and DM, respectively.

\section{NAFLD Histology}

A single pathologist, blinded to the patients clinical history and laboratory tests, reviewed the slides using the nonalcoholic steatohepatitis (NASH) Clinical Network Scoring System by Kleiner and Brunt (20). In brief, the presence and severity of steatosis (0-3), lobular inflammation (0-3), hepatocyte ballooning (0-2), and fibrosis (0-4) were scored. NASH activity score $(0-8)$ is the sum of steatosis, lobular inflammation, and ballooning. Global NASH score (0-12) is the sum of NASH activity score and fibrosis.

\section{Dietary Composition, Physical Activity, and Alcohol Consumption}

The Block FFQ 98 is the most frequently used questionnaire for metabolic and dietary intervention studies and it has been used in mixed gender and multiethnic populations (17). It is a validated, self-administered questionnaire with pictures of standardized serving sizes to estimate the usual dietary intake. The questionnaire was mailed to the patients to be filled out at home and reviewed by the dietician at the time of the study visit for completeness. The dietitians inquired if the questionnaire reflected common foodstuffs participants typically consumed, and if there were any omissions or foods that were not addressed. The completed questionnaires were sent to Block Dietary Data Systems in Berkeley for analysis. The data provided information regarding total calories consumed per day, percent of carbohydrate, protein, fat, sweets, and alcohol consumption. The consumed fat was divided into monounsaturated, polyunsaturated, and saturated fat. Fiber, cholesterol, and mineral consumption were also measured. 
Paffenbarger PAQ is a well-validated questionnaire (18) designed to assess leisure physical activity. The time frame of recall can include activity during the preceding week and year at the time of administration. Leisurely activity is divided into light, moderately vigorous, and combination activities. For example, light activity ( $5 \mathrm{kcal} / \mathrm{min}$ ) includes golf, walking, gardening, carpentry, bowling, baseball, biking, boating, dancing, and ice skating. Examples of moderately vigorous activity $(10 \mathrm{kcal} / \mathrm{min})$ include swimming, tennis, squash, racquetball, jogging, running, basketball, skiing, and ice hockey. Examples of combined activity $(7.5 \mathrm{kcal} / \mathrm{min})$ include weight lifting. Physical activities were ranked (kilocalorie expenditure) by metabolic equivalent (MET) value. Using this information the average kilocalories burned in a week was estimated and used for analysis.

Skinner survey, a well-validated instrument that estimates alcohol consumption throughout an individual's lifetime, was used to interview patients and determine the amount of alcohol consumed (21). The information obtained included the style of drinking, type of alcoholic beverage, average and maximum amount drank each time, and life events that may have affected drinking patterns.

\section{Statistical Analysis}

Comparisons between those with and without metabolic syndrome were performed using paired $t$-test and $\chi^{2}$ analyses as appropriate (continuous variables using a two-sample $t$-test and categorical variables using $\chi^{2}$ test). Correlation between histology and variables was analyzed by Pearson's correlation coefficient. Statistical analysis was performed using SAS 9.1 (SAS Institute Inc., Cary, NC).

\section{RESULTS}

Ninety-one patients with histologically confirmed NAFLD were evaluated for this study. Baseline clinical, biochemi$\mathrm{cal}$, and histological characteristics of these 91 patients are shown in Table 1. Thirty-one (34\%) patients met criteria for metabolic syndrome; 75 patients $(82 \%)$ had evidence of IR defined as HOMA index $\geq 2.0$. Diabetes, hypertension, and hyperlipidemia were seen in $18(19.8 \%), 43(47.3 \%)$, and $49(53.9 \%)$ patients, respectively. None of the patients had weight loss surgery in the past. Overall, histologic disease severity of NAFLD was mild with mean NASH activity, fibrosis, and global scores of 3.0, 1.5, and 4.9, respectively.

The mean total kilocalories consumed per day were 1,866 \pm 762.5 with the average proportion of dietary carbohydrate, fat, and protein consumption of $47 \%, 38 \%$, and $15 \%$, respectively. Men consumed an average of 1,984 $\pm 779 \mathrm{kcal} /$ day and women consumed $1,776 \pm 745 \mathrm{kcal} /$ day $(p=0.20)$. The total amount of calories consumed tended to be lower in the group of patients with metabolic syndrome. Analysis of the daily average dietary macronutrient intake based on gender showed that consumption of carbohydrate, fat, and protein was $45 \%, 40 \%$, and $15 \%$ of total daily intake among men and $49 \%, 37 \%$, and $15 \%$ among women, respectively, in our study $(p=0.64,0.06,0.28)$. The only dietary difference between men and women was that men had a higher consumption of saturated fat than women ( $27 \mathrm{~g} /$ day $v s 21 \mathrm{~g} /$ day, $p=0.01)$. Average leisurely physical activity was $1,325 \mathrm{kcal} / \mathrm{wk}$ with no significant difference seen between men and women.

Table 1 compares the clinical, biochemical, and histological characteristics of NAFLD patients with and without metabolic syndrome. There was no statistically significant difference in gender distribution, BMI, ALT, and AST levels between patients with and without metabolic syndrome. As can be expected, patients with metabolic syndrome had significantly greater mean waist circumference and waist-hip ratio, lower HDL cholesterol, and higher triglyceride levels compared with those without metabolic syndrome. In addition, patients with metabolic syndrome also had a greater degree of IR.

\section{Dietary Composition in NAFLD Subjects with and without Metabolic Syndrome}

Patients with metabolic syndrome had significantly higher mean carbohydrate consumption (percent of calories, $51 \pm$ 11 vs $45 \pm 10, p=0.03$ ) than those without metabolic syndrome. In addition, the subjects with metabolic syndrome also consumed less fat (percent of calories, $34 \pm 10 \mathrm{vs} 40 \pm$ $8, p=0.01$ ). When the different types of fat consumption, including saturated, polyunsaturated, and monounsaturated, were examined, there was no significant difference between the two groups. Although there was a tendency for those without metabolic syndrome to consume more calories and fiber $(17.7 \pm 9.4 v s 16.2 \pm 6.9 \mathrm{~g} /$ day, $p=0.4)$, this was not statistically significant. In addition, percent of calories from protein did not differ between those with and without metabolic syndrome (Fig. 1).

\section{Physical Activity in NAFLD Subjects with and without Metabolic Syndrome}

Leisurely physical activity, measured as kilocalories per week, did not significantly differ between patients with and without metabolic syndrome. Using the median physical activity value of $1,036 \mathrm{kcal} / \mathrm{wk}$, patients were divided into two groups. There was no difference in the waist circumference, waist/hip ratio, HOMA, histologic severity of NAFLD, or proportion with metabolic syndrome between these two groups. However, BMI was lower in the group that exercised more than $1,036 \mathrm{kcal} / \mathrm{wk}(34.7 \pm 6.8$ vs $31 \pm 6.3, p=0.01)$ (Table 1).

\section{NAFLD Histology in Subjects with and without Metabolic Syndrome}

Patients with metabolic syndrome had significantly higher mean scores for steatosis $(2.0 \pm 0.8 v s 1.37 \pm 1, p=0.002)$, NASH activity $(4.13 \pm 1.4 v s 3.13 \pm 1.4, p=0.004)$, and global NASH scores $(5.9 \pm 1.7 v s 4.4 \pm 2.3, p=0.0006)$. Although there was a trend toward a higher mean fibrosis score in patients with metabolic syndrome $(1.8 \pm 1.3$ vs 1.29 \pm 1.1 ), this was not statistically significant (Table 1 ). 
Table 1. Characteristics of NAFLD Patients with and without Metabolic Syndrome

\begin{tabular}{|c|c|c|c|c|}
\hline & $\begin{array}{l}\text { All Patients } \\
(\mathrm{N}=91)\end{array}$ & $\begin{array}{l}\text { Metabolic Syndrome } \\
(\mathrm{N}=31)\end{array}$ & $\begin{array}{l}\text { No Metabolic Syndrome } \\
\qquad(\mathrm{N}=60)\end{array}$ & $\begin{array}{c}p \text { Value } \\
(\mathrm{MS} v s \text { No MS) }\end{array}$ \\
\hline \multicolumn{5}{|l|}{ Clinical and biochemical features } \\
\hline Age (yr) & $47.6+11.8$ & $47 \pm 14$ & $48 \pm 10$ & 0.87 \\
\hline Gender (Female \%) & 57 & 39 & 45 & 0.66 \\
\hline $\operatorname{BMI}\left(\mathrm{kg} / \mathrm{m}^{2}\right)$ & $32.8 \pm 6.8$ & $34.3 \pm 4.2$ & $32.0 \pm 7.7$ & 0.08 \\
\hline $\mathrm{BMI} \geq 30(\%)$ & 42 & 84 & 53 & 0.008 \\
\hline $\mathrm{BMI} \geq 35(\%)$ & 25 & 39 & 20 & 0.09 \\
\hline Waist circumference (inches) & $36.4 \pm 11.2$ & $39.5 \pm 9.5$ & $34.8 \pm 11.8$ & 0.04 \\
\hline Truncal obesity $(\%)^{*}$ & 8 & 13 & 5 & 0.22 \\
\hline Waist-hip ratio & $0.9 \pm 0.1$ & $0.95 \pm 0.07$ & $0.92 \pm 0.07$ & 0.03 \\
\hline Systolic BP (mmHg) & $129 \pm 16$ & $131.2 \pm 18$ & $128.5 \pm 14.7$ & 0.45 \\
\hline Diastolic BP (mmHg) & $76 \pm 10$ & $76.7 \pm 9.5$ & $75.9 \pm 10.4$ & 0.70 \\
\hline $\mathrm{BP} \geq 135 / 85(\%)$ & 32 & 42 & 27 & 0.21 \\
\hline Total cholesterol (mg/dL) & $203.9 \pm 47.7$ & $213.7 \pm 56.6$ & $198.81 \pm 41.8$ & 0.20 \\
\hline LDL cholesterol (mg/dL) & $115+41$ & $120+46$ & $113+39$ & 0.45 \\
\hline HDL cholesterol (mg/dL) & $50 \pm 15$ & $41.7 \pm 11.3$ & $54.5 \pm 15.0$ & 0.0094 \\
\hline $\mathrm{HDL} \leq 50(\%)$ & 52 & 77 & 38 & 0.002 \\
\hline Fasting TG (mg/dL) & $192.1 \pm 182.4$ & $273.6 \pm 262.1$ & $149.3 \pm 100.6$ & 0.016 \\
\hline $\mathrm{TG} \geq 150(\%)$ & 71 & 84 & 32 & $<0.0001$ \\
\hline Fasting insulin $(\mu \mathrm{U} / \mathrm{mL})$ & $20.29 \pm 11.28$ & $24.7 \pm 9.1$ & $17.6 \pm 11.3$ & 0.002 \\
\hline Fasting glucose (mg/dL) & $101 \pm 24.7$ & $106.1 \pm 0.3$ & $98.4 \pm 25.0$ & 0.16 \\
\hline Glucose $\geq 110(\%)$ & 19 & 29 & 13 & 0.12 \\
\hline HOMA index & $5.2 \pm 3.6$ & $6.7 \pm 3.5$ & $4.4 \pm 3.5$ & 0.005 \\
\hline ALT (IU/dL) & $78.2 \pm 51.5$ & $79.2 \pm 51.8$ & $77.7 \pm 51.8$ & 0.90 \\
\hline AST (IU/dL) & $54 \pm 36$ & $59.9 \pm 37.4$ & $50.72+35.1$ & 0.26 \\
\hline Hx of DM (\%) & 19 & 29 & 13 & 0.12 \\
\hline Hx HTN $(\%)$ & 47 & 71 & 35 & 0.002 \\
\hline Hx hyperlipidemia (\%) & 54 & 65 & 48 & 0.21 \\
\hline Calories/day (FFQ 98) & $1,866 \pm 762.5$ & $1,795 \pm 722$ & $1,903 \pm 787$ & 0.51 \\
\hline Physical activity (PAQ, kcal/wk) & $1,325.7 \pm 1,274$ & $1,149 \pm 1,109$ & $1,417 \pm 1,351$ & 0.36 \\
\hline \multicolumn{5}{|c|}{$\begin{array}{l}\text { NAFLD histology according to NASH-Clinical } \\
\text { Network scoring system }\end{array}$} \\
\hline Steatosis score $(0-3)$ & $1.6 \pm 1.0$ & $2.0 \pm 0.8$ & $1.37 \pm 1$ & 0.002 \\
\hline Lobular inflammation $(0-3)$ & $1.4 \pm 0.8$ & $1.5 \pm 0.8$ & $1.4 \pm 0.8$ & 0.70 \\
\hline Hepatocyte ballooning $(0-2)$ & $0.5 \pm 0.5$ & $0.6 \pm 0.5$ & $0.4 \pm 0.5$ & 0.008 \\
\hline NASH activity score $(0-8)$ & $3.5 \pm 1.7$ & $4.1 \pm 1.4$ & $3.1 \pm 1.7$ & 0.004 \\
\hline Fibrosis score $(0-4)$ & $1.5 \pm 1.2$ & $1.8 \pm 1.3$ & $1.29 \pm 1.1$ & 0.06 \\
\hline Global NASH score $(0-12)$ & $4.9 \pm 2.2$ & $5.9 \pm 1.7$ & $4.4 \pm 2.3$ & 0.0006 \\
\hline
\end{tabular}

$\mathrm{NAFLD}=$ nonalcoholic fatty liver disease $\mathrm{BMI}=$ body mass index $\mathrm{BP}=$ blood pressure $\mathrm{LDL}=$ low density lipoprotein; $\mathrm{HDL}=$ high density lipoprotein; TG $=$ triglyceride; HOMA = homeostasis model assessment; ALT = alanine aminotransferase; AST = aspartate aminotransferase; DM = diabetes mellitus; HTN = hypertension; FFQ = food frequency questionnaire; $\mathrm{PAQ}=$ physical activity questionnaire; $\mathrm{NASH}=$ nonalcoholic steatohepatitis; MS $=$ metabolic syndrome.

*By Fishers exact test.

\section{Biochemical and Anthropometric Differences in Patients with High Versus Low Global NASH Score}

The median global NASH score was 5 . When patients with low and high global NASH score $(0-5$ vs 6-12) were compared, the group with higher global NASH score had a greater proportion of patients with obesity $(\mathrm{BMI}>30)$ and metabolic syndrome, higher levels of fasting insulin, HOMA index, serum AST levels, and less physical activity $(p=0.01-$ 0.001) (Table 2).

\section{The Effect of Diet Composition and Physical Activity Was Further Assessed}

Factors associated with NASH severity (high $v s$ low global NASH score) were analyzed by univariable, followed by multivariable, regression models. In univariable analysis, factors associated with a low global NASH score were male gender and physical activity (kcal/wk) and those associated with high global NASH score were IR (HOMA index) and the presence of metabolic syndrome. In multivariable analysis, the pres- ence of metabolic syndrome was significantly associated with high global NASH score after adjusting for all other significant factors (OR 3.48, 95\% CI 1.22-9.85, $p=0.019$ ). Factors other than metabolic syndrome that were independently associated with a NASH severity included female gender (OR $0.28,95 \%$ CI $0.09-0.9, p=0.035$ ) and HOMA index (OR $1.27,95 \%$ CI $1.05-1.54, p=0.014)$.

\section{Alcohol Consumption}

Neither current or lifetime alcohol consumption was related to histologic severity of NAFLD (data not shown). In addition, there was no difference in alcohol consumption between patients with and without metabolic syndrome.

\section{DISCUSSION}

This study compares the dietary intake, physical activity, and liver histology in patients with and without metabolic syndrome. The prevalence of metabolic syndrome in our patients 
Table 2. Comparison of Patients with High versus Low Global NASH Score

\begin{tabular}{|c|c|c|c|}
\hline & $\begin{array}{c}\text { Global NASH } 0-5 \\
(\mathrm{~N}=56)\end{array}$ & $\begin{array}{c}\text { Global NASH } 6-12 \\
(\mathrm{~N}=35)\end{array}$ & $p$ Value \\
\hline Age (yr) & $48 \pm 11$ & $47 \pm 13$ & 0.92 \\
\hline Gender (female \%) & 48 & 29 & 0.05 \\
\hline $\operatorname{BMI}\left(\mathrm{kg} / \mathrm{m}^{2}\right)$ & $32 \pm 8$ & $34 \pm 5$ & 0.08 \\
\hline $\mathrm{BMI} \geq 30(\%)^{*}$ & 52 & 83 & 0.006 \\
\hline $\mathrm{BMI} \geq 35(\%)$ & 25 & 37 & 0.32 \\
\hline Waist circumference (inches) & $35.5 \pm 11.3$ & $39 \pm 10.9$ & 0.20 \\
\hline Truncal obesity $(\%)^{*}$ & 36 & 49 & 0.65 \\
\hline Waist-hip ratio & $0.93 \pm 0.07$ & $0.94 \pm 0.08$ & 0.67 \\
\hline Systolic BP (mmHg) & $129 \pm 17$ & $130 \pm 14$ & 0.78 \\
\hline Diastolic BP (mmHg) & $76 \pm 9$ & $76 \pm 12$ & 0.82 \\
\hline $\mathrm{BP} \geq 135 / 85(\%)$ & 23 & 43 & 0.08 \\
\hline Total cholesterol (mg/dL) & $201 \pm 45$ & $208 \pm 53$ & 0.49 \\
\hline HDL cholesterol (mg/dL) & $60 \pm 57$ & $47 \pm 16$ & 0.14 \\
\hline $\mathrm{HDL} \leq 50(\%)$ & 54 & 66 & 0.36 \\
\hline Fasting TG (mg/dL) & $167 \pm 107$ & $231 \pm 257$ & 0.17 \\
\hline $\mathrm{TG} \geq 150(\%)$ & 39 & 66 & 0.025 \\
\hline Fasting insulin $(\mu \mathrm{U} / \mathrm{dL})$ & $17 \pm 10$ & $25 \pm 12$ & 0.001 \\
\hline Fasting glucose (mg/dL) & $98 \pm 26$ & $105 \pm 23$ & 0.18 \\
\hline Glucose $\geq 110(\%)$ & 13 & 29 & 0.10 \\
\hline Hx of DM $v s$ no DM (\%) & 20 & 20 & 0.82 \\
\hline Hx of HTN vs no HTN (\%) & 9 & $3: 32(9)$ & $1.00^{*}$ \\
\hline Hx of hyperlipidemia (\%) & 56 & 54 & 0.88 \\
\hline HOMA index & $4.3 \pm 3.1$ & $6.8 \pm 3.9$ & 0.003 \\
\hline Metabolic syndrome (\%) & 21 & 54 & 0.003 \\
\hline ALT $(\mathrm{IU} / \mathrm{dL})$ & $97 \pm 45$ & $94 \pm 66$ & 0.05 \\
\hline AST $(\mathrm{IU} / \mathrm{dL})$ & $46 \pm 24$ & $66 \pm 47$ & 0.03 \\
\hline \multicolumn{4}{|c|}{ Dietary macronutrient composition } \\
\hline Kilocalories/day (FFQ) & $1,859 \pm 805$ & $1,877 \pm 701$ & 0.91 \\
\hline$\%$ Carbohydrate & $46 \pm 11$ & $50 \pm 11$ & 0.14 \\
\hline$\%$ Fat & $39 \pm 9$ & $37 \pm 10$ & 0.16 \\
\hline & $16 \pm 3$ & $15 \pm 3$ & 0.44 \\
\hline \multicolumn{4}{|l|}{ Physical activity } \\
\hline $\mathrm{kcal} / \mathrm{wk}(\mathrm{PAQ})$ & $1,566 \pm 1,429$ & $942 \pm 864$ & 0.01 \\
\hline
\end{tabular}

$\mathrm{NASH}=$ nonalcoholic steatohepatitis; $\mathrm{BMI}=$ body mass index; $\mathrm{BP}=$ blood pressure; $\mathrm{HDL}=$ high density lipoprotein; $\mathrm{TG}=$ triglyceride; Hx $=$ history of; $\mathrm{DM}=$ diabetes mellitus; HTN = hypertension; HOMA = homeostasis model assessment; ALT = alanine aminotransferase; AST = aspartate aminotransferase; FFQ = food frequency questionnaire; $\mathrm{PAQ}=$ physical activity questionnaire.

*B By Fisher's exact test.

is similar to that reported in other studies in NAFLD $(36 \%$ of NAFLD patients without diabetes) (22), which is higher than that of the general population in the United States. As expected, IR was significantly greater in the group with metabolic syndrome. We found that NAFLD patients with metabolic syndrome consumed more carbohydrates, less fat, and equal amounts of protein and total calories, compared with those without metabolic syndrome. Physical activity did not differ between the two groups. When comparing histologic severity of NAFLD, patients with metabolic syndrome had higher scores for steatosis grade, NASH activity, and global NASH severity.

Despite the increasing prevalence of NAFLD in the United States there is no effective medical therapy available to date. Current recommendations are to lose weight through diet and exercise with no robust evidence to support this recommendation (23). Individuals who are physically active and consume a low carbohydrate diet have a lower risk of having metabolic syndrome $(5,12,24)$. In addition, obese patients have improved IR (25-29), abdominal obesity (25), and lower odds for hepatic inflammation (14) with a lower carbohydrate diet. We have recently shown that intense nutritional counseling with dietary modification focusing on a diet improving IR and decreasing carbohydrate consumption, results in histologic improvement in NASH (30). Although a low-fat diet has generally been recommended in patients with NAFLD, recent studies show that a greater short-term weight loss and improvement in markers of the metabolic syndrome is associated with a low-carbohydrate diet without significant adverse effects (31). The role of dietary composition with a decrease in carbohydrate relative to the total calories consumed, as well as increased physical activity, in NAFLD histology, however, needs to be further studied.

Patients with a higher global NASH score were more likely to be obese, had greater IR as measured by HOMA, higher AST, and less physical activity. Our findings are consistent with previous studies suggesting that patients with NAFLD and DM, which is a component of metabolic syndrome and associated with IR, have more aggressive disease with a higher percentage of cirrhosis and higher odds of liver cell necrosis compared with those without diabetes (32). Patients who have greater splanchnic (visceral) fat distribution may be particularly prone to the effects of IR and possibly to a more aggressive form of NASH $(6,9)$. This may explain the 


\section{All patients $(\mathrm{N}=91)$

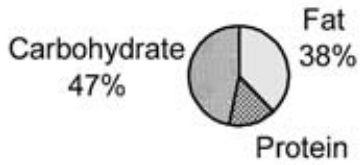 \\ A \\ $15 \%$}

\section{Patients with metabolic syndrome $(\mathrm{N}=31)$}

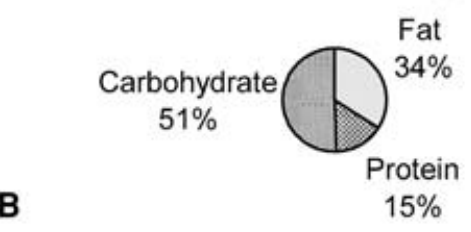

\section{Patients without metabolic syndrome $(\mathrm{N}=60)$}

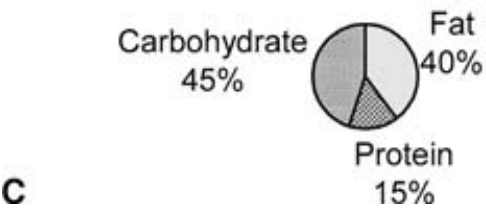

Figure 1. Macronutrient composition of diet in NAFLD patients. $B$ versus $C$ carbohydrate, fat, and protein $p$ value $=0.03,0.006$, and 0.91 , respectively.

more severe disease seen in our patients with metabolic syndrome and higher global NASH score, who had significantly larger waist circumference (a measure of visceral adiposity) and a greater degree of IR. When controlled for other factors, including dietary composition and physical activity, the presence of metabolic syndrome appeared to be one of the most significant risk factors for global NASH severity in addition to HOMA index and female gender.

The time of NAFLD diagnosis to enrollment in the study was less than 4 wk. Physical activity and dietary information obtained was an average over the past year; therefore, the information obtained for dietary food consumption and physical activity was likely a good reflection of the patient's dietary and exercise habits prior to the diagnosis of NAFLD.

Our study does have limitations. Patients with ALT $<1.5$ times the upper limit of normal were excluded if they did not have radiologic imaging with features of steatosis, thus the patients enrolled do not represent all patients with NAFLD. The size of the study was not large, all patients were from a single institution, and NAFLD histologic severity was limited to a mean global NASH score of $4.9 \pm 2.2$ and did not represent the entire spectrum of NAFLD. The lack of a control group does not allow us to establish an absolute modification of dietary habits on histologic severity.

Dietary and physical activity information was obtained through self-reported questionnaires, which may be subject to underreporting and recall bias. The dietary questionnaire assessed the dietary intake of diverse food groups and snacks based on a typical American diet. The majority of the patients in our study were Caucasian Americans and based on the dietician interviews, this questionnaire was appropriate in capturing their usual dietary intake. The dietary questionnaire was able to capture information regarding saturated, monounsaturated, and polyunsaturated fat but we were not able to obtain trans fat consumption, which may affect IR and NAFLD histology. Though it has been shown that a carbohydrate-rich diet, especially with a high glycemic index, worsens IR (26), the FFQ 98 does not assess the glycemic index of carbohydrates.

The PAQ is a reflection of leisurely activities, blocks walked, and flights of stairs climbed. Many of the physical activities noted by homemakers are from household activities that this questionnaire does not address. With the compendium of physical activities (33) that facilitates coding of physical activities, we are able to better estimate the MET used for each activity and this may be a better estimate of activity in the future.

In conclusion, we have shown that those with metabolic syndrome and NAFLD have greater histologic severity of their underlying liver disease than patients without metabolic syndrome. In addition, patients with metabolic syndrome and NAFLD also consume more carbohydrates and less fat compared with those without metabolic syndrome. Further studies of a larger number of NAFLD patients with a broad spectrum of histologic severity will be needed to determine the effect of dietary modification such as carbohydrate restriction on histologic improvement of NAFLD.

\section{ACKNOWLEDGMENTS}

This research was conducted at the University of Michigan General Clinical Research Center (GCRC) with funding from the GCRC (GCRC Grant \#M01-RR0004).

\section{STUDY HIGHLIGHTS}

\section{What Is Current Knowledge}

- Nonalcoholic fatty liver disease (NAFLD) is a manifestation of metabolic syndrome.

- A diet with fewer carbohydrates and exercise improves metabolic syndrome.

- NAFLD patients with metabolic syndrome consume a diet with fewer carbohydrates and more fat compared to NAFLD patients without metabolic syndrome.

- NAFLD patients with metabolic syndrome have more severe histologic findings of NAFLD compared to those without metabolic syndrome.

\section{What Is New Here}

- We have shown that there may be a role for a diet with fewer carbohydrates and moderate fat consumption in improving NAFLD histology in patients with NAFLD and metabolic syndrome. 
Reprint requests and correspondence: Hari Conjeevaram, M.D., M.S., Division of Gastroenterology, The University of Michigan, 3912 Taubman Center, 1500 East Medical Center Drive, Ann Arbor, MI 48109-0362.

Received December 5, 2005; accepted April 5, 2006.

\section{REFERENCES}

1. Clark JM, Brancati FL, Diehl AM. The prevalence and etiology of elevated aminotransferase levels in the United States. Am J Gastroenterol 2003;98:960-7.

2. Angulo P. Nonalcoholic fatty liver disease. N Engl J Med 2002;346:1221-31.

3. Ruhl CE, Everhart JE. Determinants of the association of overweight with elevated serum alanine aminotransferase activity in the United States. Gastroenterology 2003;124:71-9.

4. Ford ES, Giles WH, Dietz WH. Prevalence of the metabolic syndrome among US adults: Findings from the third National Health and Nutrition Examination Survey. JAMA 2002;287:356-9.

5. Yao M, Lichtenstein AH, Roberts SB, et al. Relative influence of diet and physical activity on cardiovascular risk factors in urban Chinese adults. Int J Obes Relat Metab Disord 2003;27:920-32.

6. Pagano G, Pacini G, Musso G, et al. Nonalcoholic steatohepatitis, insulin resistance, and metabolic syndrome: Further evidence for an etiologic association. Hepatology 2002;35:367-72.

7. Marchesini G, Brizi M, Bianchi G, et al. Nonalcoholic fatty liver disease: A feature of the metabolic syndrome. Diabetes 2001;50:1844-50.

8. Day CP, James OF. Steatohepatitis: A tale of two "hits"? Gastroenterology 1998;114:842-5.

9. Sanyal AJ, Campbell-Sargent C, Mirshahi F, et al. Nonalcoholic steatohepatitis: Association of insulin resistance and mitochondrial abnormalities. Gastroenterology 2001;120:1183-92.

10. DeFronzo RA, Ferrannini E. Insulin resistance. A multifaceted syndrome responsible for NIDDM, obesity, hypertension, dyslipidemia, and atherosclerotic cardiovascular disease. Diabetes Care 1991;14:173-94.

11. Eckel RH, Grundy SM, Zimmet PZ. The metabolic syndrome. Lancet 2005;365:1415-28.

12. Arora SK, McFarlane SI. The case for low carbohydrate diets in diabetes management. Nutr Metab (Lond) 2005;2:16-24.

13. Thompson PD, Buchner D, Pina IL, et al. Exercise and physical activity in the prevention and treatment of atherosclerotic cardiovascular disease: A statement from the Council on Clinical Cardiology (Subcommittee on Exercise, Rehabilitation, and Prevention) and the Council on Nutrition, Physical Activity, and Metabolism (Subcommittee on Physical Activity). Circulation 2003;107:3109-16.

14. Solga S, Alkhuraishe AR, Clark JM, et al. Dietary composition and nonalcoholic fatty liver disease. Dig Dis Sci 2004;49:1578-83.

15. Matthews DR, Hosker JP, Rudenski AS, et al. Homeostasis model assessment: Insulin resistance and beta-cell function from fasting plasma glucose and insulin concentrations in man. Diabetologia 1985;28:412-9.

16. Yokoyama H, Emoto M, Fujiwara S, et al. Quantitative insulin sensitivity check index and the reciprocal index of homeostasis model assessment in normal range weight and moderately obese type 2 diabetic patients. Diabetes Care 2003;26:2426-32.

17. Block G, Woods M, Potosky A, et al. Validation of a selfadministered diet history questionnaire using multiple diet records. J Clin Epidemiol 1990;43:1327-35.

18. Paffenbarger RS Jr, Wing AL, Hyde RT. Physical activity as an index of heart attack risk in college alumni. Am J Epidemiol 1978;108:161-75.

19. Ford ES, Giles WH. A comparison of the prevalence of the metabolic syndrome using two proposed definitions. Diabetes Care 2003;26:575-81.

20. Kleiner DE, Brunt EM, Van Natta M, et al. Design and validation of a histological scoring system for nonalcoholic fatty liver disease. Hepatology 2005;41:1313-21.

21. Skinner HA, Sheu WJ. Reliability of alcohol use indices. The lifetime drinking history and the MAST. J Stud Alcohol 1982;43:1157-70.

22. Marchesini G, Bugianesi E, Forlani G, et al. Nonalcoholic fatty liver, steatohepatitis, and the metabolic syndrome. Hepatology 2003;37:917-23.

23. Clark JM. Weight loss as a treatment for nonalcoholic fatty liver disease. J Clin Gastroenterol 2006;40(suppl 1):S39-43.

24. Zhu S, St-Onge MP, Heshka S, et al. Lifestyle behaviors associated with lower risk of having the metabolic syndrome. Metabolism 2004;53:1503-11.

25. LaHaye SA, Hollett PM, Vyselaar JR, et al. Comparison between a low glycemic load diet and a Canada Food Guide diet in cardiac rehabilitation patients in Ontario. Can J Cardiol 2005;21:489-94.

26. Layman DK, Boileau RA, Erickson DJ, et al. A reduced ratio of dietary carbohydrate to protein improves body composition and blood lipid profiles during weight loss in adult women. J Nutr 2003;133:411-7.

27. Luscombe-Marsh ND, Noakes M, Wittert GA, et al. Carbohydrate-restricted diets high in either monounsaturated fat or protein are equally effective at promoting fat loss and improving blood lipids. Am J Clin Nutr 2005;81:76272.

28. Noakes M, Keogh JB, Foster PR, et al. Effect of an energyrestricted, high-protein, low-fat diet relative to a conventional high-carbohydrate, low-fat diet on weight loss, body composition, nutritional status, and markers of cardiovascular health in obese women. Am J Clin Nutr 2005;81:1298306.

29. Farnsworth E, Luscombe ND, Noakes M, et al. Effect of a high-protein, energy-restricted diet on body composition, glycemic control, and lipid concentrations in overweight and obese hyperinsulinemic men and women. Am J Clin Nutr 2003;78:31-9.

30. Huang MA, Greenson JK, Chao C, et al. One-year intense nutritional counseling results in histological improvement in patients with non-alcoholic steatohepatitis: A pilot study. Am J Gastroenterol 2005;100:1072-81.

31. Gill HK, Wu GY. Non-alcoholic fatty liver disease and the metabolic syndrome: Effects of weight loss and a review of popular diets. Are low carbohydrate diets the answer? World J Gastroenterol 2006;12:345-53.

32. Younossi ZM, Gramlich T, Matteoni CA, et al. Nonalcoholic fatty liver disease in patients with type 2 diabetes. Clin Gastroenterol Hepatol 2004;2:262-5.

33. Ainsworth BE, Haskell WL, Whitt MC, et al. Compendium of physical activities: An update of activity codes and MET intensities. Med Sci Sports Exerc 2000;32:S498-504.

\section{CONFLICT OF INTEREST}

Guarantor of the article: Hari S. Conjeevaram, M.D., M.S., and Helen Kang, M.D.

Financial support: General Clinical Research Center Grant \#M01-RR0004

Potential competing interests: None 